Revue d'histoire des chemins de fer

Le chemin de fer à la conquête des campagnes

\title{
Clôture du colloque
}

\section{François Gerbaud}

\section{OpenEdition}

\section{Journals}

Édition électronique

URL : https://journals.openedition.org/rhcf/2092

DOI : 10.4000/rhcf.2092

Éditeur

Rails \& histoire

Édition imprimée

Date de publication : 2 mai 2002

Pagination : 406-409

ISBN : 00996-9403

ISSN : 0996-9403

\section{Référence électronique}

François Gerbaud, «Clôture du colloque », Revue d'histoire des chemins de fer [En ligne], 24-25 | 2002

mis en ligne le 28 avril 2015, consulté le 22 avril 2022. URL : http://journals.openedition.org/rhcf/2092 ; DOI : https://doi.org/10.4000/rhcf.2092 


\section{François GERBAUD}

\section{Clôture du colloque}

Mesdames et Messieurs de l'Association pour l'histoire des chemins de fer en France, Monsieur le Professeur Wolkowitsch, voici que, sur les rails de l'évocation des souvenirs de ce que j’appellerai la mémoire positive, votre colloque arrive à son terminus en ce chef-lieu du département de l'Indre, partie historique du Cœur de France.

Parlementaire très attaché au ferroviaire et à son évolution, je suis très honoré de vous y saluer, sans avoir à vous demander par les hautparleurs de notre amitié reconnaissante si vous n'avez rien oublié dans les voitures du convoi de notre voyage au Pays d'hier.

Très honoré surtout de vous remercier d'avoir choisi le Centre universitaire de Châteauroux pour nous y inviter scientifiquement à nous pencher ensemble sur ce que j'appellerai un passé actuel, tant il est vrai que le chemin de fer est à l'aube de ce XXI ${ }^{e}$ siècle appeler à jouer dans toutes les politiques d'aménagement du territoire, qu'elles soient régionales, nationales ou européenne, le même rôle et la même importance économique, sociale et humaine qu'au siècle qui l'a vu naitre il y a plus de 150 années, même si, comme le dit Paul Valéry : «le futur n’a plus l'habitude d'être ce qu'il était. »

Aide-mémoire prestigieux, votre neuvième colloque a donné au département de l'Indre la pertinente occasion de se souvenir de ce que furent les réseaux secondaires et de ce que, à travers eux, le chemin de fer a apporté de réalité économique, sociale et humaine à nos campagnes.

Les évidences étant souvent au seuil de l'oubli, votre colloque a été un rappel très important de ce que les petites lignes secondaires d'hier ont eu d'essentiel dans la vie de nos concitoyens et dans leur progrès social et, disant cela, j'emprunte au professeur Wolkowitch l'histoire des sabots siciliens.

C'est particulièrement vrai pour notre département ; de nombreuses lignes aujourd'hui disparues ont irrigué le territoire berrichon avant les autobus et les autocars, créant d'innombrables liens de communication, de vie et d'échange, des interconnexions nombreuses avec 
la ligne Paris-Toulouse. Cette liaison fut, en effet, le premier maillon d'un réel désenclavement, et elle en reste un élément capital pour tous les départements du Centre et du Sud.

Ce rôle, le POLT - liaison Paris-Orléans-Limoges-Toulouse dont la rénovation décidée, annoncée, programmée et financée jusqu’à son aboutissement en 2004 va naturellement le pérenniser et le renforcer : il fera de cet axe ferroviaire historique l'un des axes nord-sud à vocation nationale et européenne, permettant ainsi au pendulaire d'innover sur une grande partie de notre territoire.

Cette liaison, modernisée à l'heure ou va s'accroître le nombre de voyageurs et où se pose l'urgente nécessité de transférer sur le rail le trop-plein de marchandises circulant sur la route, est bien un élément de première importance dans une cohérente et solidaire politique d'aménagement du territoire français.

Telle a toujours été notre conviction et tel fut le sens des combats que nous n'avons jamais cessé de mener pour qu'il en soit ainsi.

Ce ne fut pas toujours facile car il a fallu beaucoup convaincre, notamment ces esprits qui, prisonniers de l'inutile et dérisoire débat entre le ballast et le bitume, entendaient donner la priorité à l'autoroute sur le rail qu'elle longe : page heureusement tournée sur l'évidence du fait que, dans une multimodalité moderne, les deux modes de transport sont complémentaires plus que concurrentiels ; vision réaliste des choses, si l'on sait que, faute de faire passer sur le rail l'accroissement des volumes de marchandises, on va en 2010 trouver chaque jour 10000 camions à la frontière espagnole comme l'a pronostiqué Claude Martinand.

Si, dans ce débat, nous avons fait preuve d'une si grande pugnacité, c'est peut-être parce que dans notre mémoire collective, les réseaux dits secondaires nous avaient donné la mesure et l'importance du rail local, qu'ils fussent tramways ou trains d'hier.

Enfin, à un titre personnel, je suis heureux que votre colloque m'ai permis d'entendre siffler le train au moins une quatrième fois.

La première fois que j'entendis siffler le train, c'était il y a déjà de nombreuses années : né à deux pas de la gare de Châteauroux, les superbes locomotives à vapeur qui déjà mettaient Paris à quatre heures de Châteauroux ont bercé dans leurs sifflements mes premiers sommeils sans pour autant les perturber.

La seconde fois, et vous en avez actualisé le souvenir, ce fut le jour où, avec mes parents sur la plate-forme d'un wagon du petit train Châteauroux-Valençay, je vis défiler la campagne berrichonne sur le 
canton que j’ai représenté pendant 31 ans à l'Assemblée départementale. C'était, dans les années 1930, un petit train dont je garde le précieux et émouvant souvenir du légendaire essoufflement lorsque, toute vapeur rageusement crachée, il grimpait la dure pente de l'avenue de Déols.

La troisième fois que $j$ 'entendis le train siffler ce fut à la fin de 1996 et au début de 1997 lorsque, au Sénat, m’échut la lourde et délicate mission d'être en première lecture le rapporteur de la loi qui, en application de l'impérieuse directive européenne 91-440, exigeait la réforme de la SNCF en séparant le réseau ferroviaire aujourd'hui propriété de RFF de son exploitation par l'actuelle SNCF. Ce fut une mission délicate que de mener à son terme et sans heurts une réforme qui fut une véritable révolution culturelle pour une entreprise à laquelle l'histoire, la technicité et la légende avaient donné tant de lustre. Une évolution cependant indispensable au maintien d'un service public en liberté surveillée par une Europe toujours en mal de directives, directives dont certaines qui sont, comme on dit, «dans les tuyaux » peuvent être source de grandes difficultés et de conflits sociaux.

Si je me permets d'évoquer devant vous, qui êtes des acteurs et des observateurs éminents du monde ferroviaire, cet aspect des choses, c'est au nom de plusieurs convictions que je n'ai pas bien sûr la vanité d'ériger en certitudes.

Je suis d'abord convaincu que, succédant aux nombreuses lois que vous avez évoquées et qui ont rythmé la marche du temps ferroviaire, la loi que j’ai rapportée, amendée et qui fut votée en termes identiques par l'Assemblée nationale était bien, avec l'aval du parlement, la meilleure réponse qui pouvait être donnée tout à la fois à la nécessaire adaptation de nos réseaux ferroviaires et aux exigences communautaires. Largement initiée par des hommes d'exceptionnelle qualité au nombre desquels je salue Claude Martinand, cette loi et son application témoignent d'un moderne réalisme.

Je suis convaincu, et je ne suis pas le seul, que, votée comme le Parlement l'avait souhaité, elle ne peut et ne doit en aucun cas être remise, même partiellement, en cause lors du premier bilan de sa mise en œuvre.

Je suis convaincu, enfin, que la régionalisation annoncée dont sept régions achèvent l'expérimentation peut être, dans l'exercice d'une responsabilité de proximité, la meilleure manière, comme le dit un dirigeant de la SNCF, de concilier dans l'intérêt général le service public et l'esprit d'entreprise - pour peu, bien sûr que l'intérêt national ne soit pas perturbé par des excès d'égoïsme régionaux générés par je ne sais quel exigeant retour sur investissement. La Région Centre où nous sommes 
est, à ce titre, exemplaire par ses investissements et ses initiatives en matière de TER comme de rénovation de gares comme elle l'est par son soutien au B.A. qui, demain, vous conduira sur une voie métrique au train pour Paris à Salbris.

S'il en est ainsi dans une paix sociale sauvegardée, la régionalisation sera, à sa manière et vous avez eu raison de le dire, le prolongement de ce que furent en leur temps les réseaux dits secondaires.

C'est en ce sens que votre colloque, où l'anecdote et l'analyse scientifique se sont heureusement mêlées, est en même temps un moment d'histoire, un signal d'avenir.

Dans un mot dont je vous prie de pardonner l'audace, j’ai dit à la tribune du Sénat : « le rail j’y crois dur comme fer!» Je ne retire rien de cette conviction car le chemin de fer, avec ses records, sa technicité sa sécurité, est bien au cœur de notre modernité galopante.

Il est à la fois, et je le souhaite tel, sans querelle entre Girondins et Jacobins. Peut-être est-il un peu moins jacobin que ne le pressentait George Sand lorsqu'elle écrivait à Lamartine dans l'autre siècle : « Les chemins de fer qui devraient, d'après un système de centralisation organisatrice, être des bienfaits inappréciables pour l'intérieur et les extrémités de la France deviendront infailliblement de nouvelles causes de destruction intellectuelle pour nos provinces ». Paris, écrivait-elle encore, « sera de plus en plus non l'entrepôt commercial de la France mais le magasin insatiable ou s'enfonceront ses produits. »

Les réseaux secondaires ont peut-être contribué à corriger celle vision un peu pessimiste de l'avenir des chemins de fer.

Merci, Mesdames et Messieurs, de ces deux journées riches de rétrospectives juridiques, techniques, sociales et géographiques.

Elles nous ramènent en cet instant au quai du constat : dans presque tous les départements les cars ont remplacé depuis longtemps déjà les petits trains et tramways d'hier et, dans nos campagnes, les abribus les gares.

La tendresse, sans doute, y a beaucoup perdu car comme le dit si bien René Char, c'est dans les gares que l'on s'embrasse le plus.

N'en concevons nulle inquiétude puisque, quoi qu'il en soit, à l'inverse de la route de fer qui fut un jour coupée, celle de l'amour ne l'est pas. 